\section{Linkage analysis combined with whole-exome sequencing identifies a novel prothrombin (F2) gene mutation in a Dutch Caucasian family with unexplained thrombosis}

Genetics play a major role in the development of venous thromboembolism (VTE). However, current thrombophilia testing identifies a known heritable defect in only a minority of VTE patients. ${ }^{1}$ A part of this missing heritability for VTE may be caused by rare variants. ${ }^{2}$ In the current study we combined genome-wide linkage analysis with whole-exome sequencing (WES) and identified a rare novel prothrombin (F2) gene mutation (c.1621C>T; p.Arg541Trp hereafter referred to as p.Arg173Trp according to chymotrypsin numbering) in a Dutch family with unexplained thrombosis.

The proband, from Dutch Caucasian ancestry, presented at the age of 31 years with a first deep vein thrombosis (DVT) during pregnancy. Shortly after, her father had DVT after hospitalization at the age of 58. The family was aware that they were prone for thrombosis, and offered a family tree of the older generations. The proband's paternal uncle had had a spontaneous pulmonary embolism at the age of 38 and 42 years, a cousin had a spontaneous DVT at the age of 44 years (data obtained from the subjects themselves, VTE diagnoses in generations I, II and III were obtained from family members). Routine thrombophilia testing in the proband was negative, excluding deficiencies of antithrombin, protein $\mathrm{C}$, protein $\mathrm{S}$, and factor $\mathrm{V}$ Leiden and the prothrombin G20210A mutation. In the years after, both the proband and her father had a spontaneous DVT recurrence, and the proband's younger sister presented with estrogenrelated pulmonary embolism. We hypothesized that this family carried a private, high risk mutation. After informed consent was obtained we included a total of 19 family members for testing.

First, genome-wide single nucleotide polymorphism genotyping was performed on 19 family members (five affected and 14 unaffected; Figure 1A and Online Supplementary Data) and identified twelve chromosomes with equally high significant linkage peaks (LOD >1). These chromosomes were: 3, 4, 7, 9, 11, 13, 14, 18, 19, 20,22 , and X. Next, WES was performed on five family members (four affected: 26101, 26103, 26105, 26206; one unaffected: 26312; Figure 1A and Online Supplementary Data). In total, 138,672 variants were detected, of which, after rigorous filtering, 113 remained common among affected family members. When we combined these results with linkage data, e.g. filtering based on their distance $(\sim 3 \mathrm{Mb})$ to the linkage peak, 30

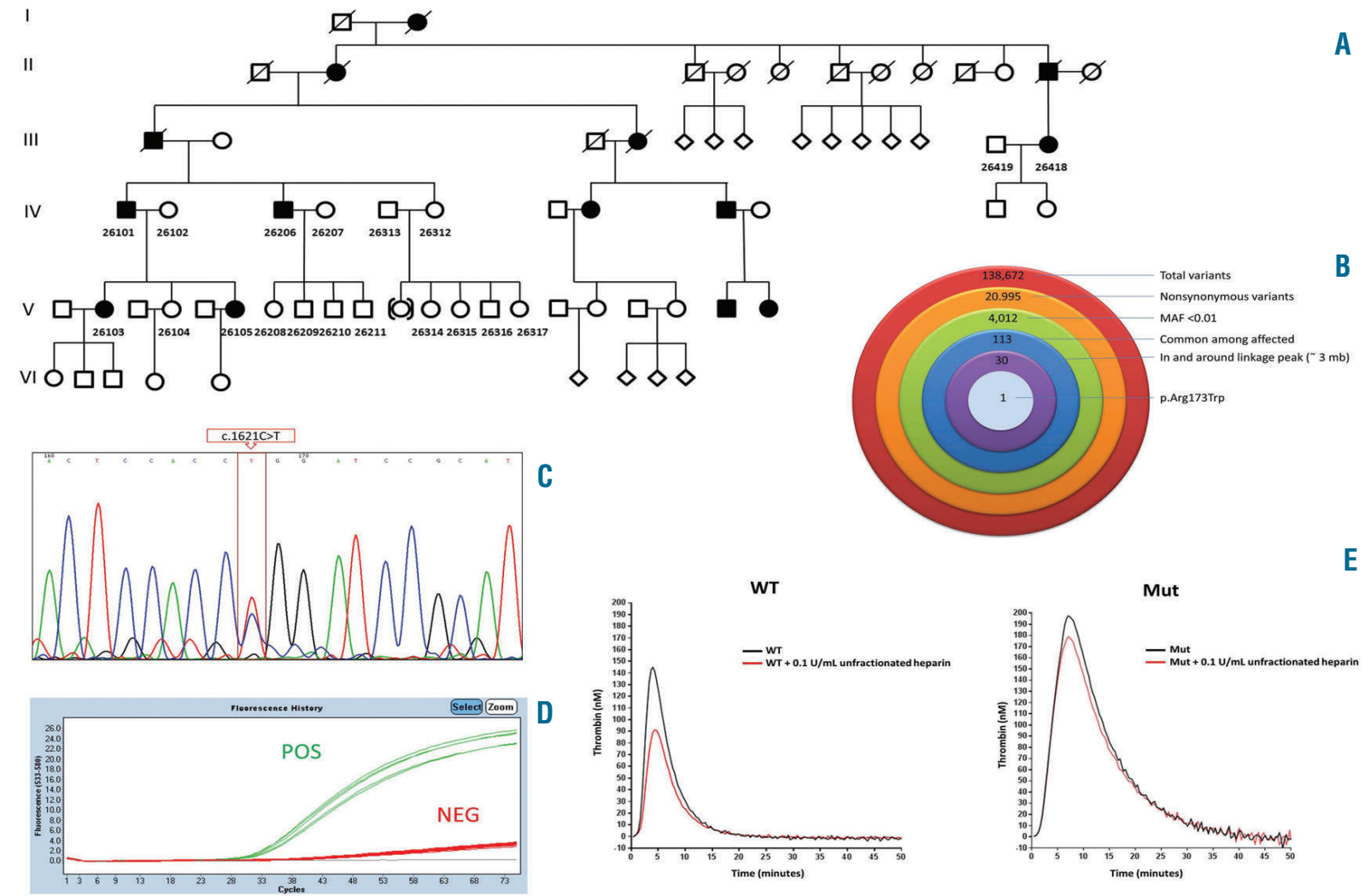

Figure 1. Identification and functional characterization of a novel prothrombin $(F 2)$ gene mutation in a Dutch family with unexplained thrombosis. (A) The pedigree. The arrow indicates the proband. Roman numbers denote the generation in the pedigree; the study number of included family members is depicted below each symbol; square symbols, male sex; round symbols, female sex; solid symbols represent VTE-affected family members, open symbols represent unaffected family members. (B) Whole-exome sequencing (WES) combined with linkage analysis. The diagram depicts effects of filtering WES data based on specific aspects including among other things such as type of mutation and location to linkage peak. (C) Direct sequencing results of exon 12 of $F 2$ gene. The prothrombin (F2) gene mutation (c.1621C>T; p.Arg173Trp) is highlighted. (D) This figure shows the genotyping results of a custom made Taqman quantitative PCR assay for 19 included family members. The green lines represent the carriers of the prothrombin (F2) gene mutation $(26101,26103,26105,26206$, and 26209$)$, whereas red lines represent the non-carriers of the mutation (26102, 26104, 26207, 26208, 26210, 26211, 26312, 26313, 26314, 26315, 26316, 26317, 26418 , and 26419). (E) Thrombin generation in prothrombin depleted plasma supplemented with wild-type (WT) and mutant protein in the presence or absence of $0.1 \mathrm{U} / \mathrm{mL}$ unfractionated heparin. 
variants remained. In the final step, in silico prediction was performed with MetaSVM and MetaLR thereby identifying 1 potentially pathogenic novel prothrombin (F2) gene mutation (p.Arg173Trp) (Figure 1B) located on chromosome 11. Using Sanger sequence analysis we confirmed the heterozygous presence of a novel prothrombin (F2) gene mutation (Figure 1C). Next, with a custom made TaqMan quantitative PCR assay (Applied Biosystems) we confirmed the presence of the prothrombin (F2) gene mutation p.Arg173Trp in four affected family members $(26101,26103,26105,26206)$, whereas one unaffected family member (216209) also carried this mutation (Figure 1D). Furthermore, one family member who did not carry the mutation (216418) had a history of DVT. Functional testing was complicated due to the use of vitamin $\mathrm{K}$ antagonists in the carriers of the prothrombin mutation with available plasma. We therefore performed functional assays with recombinant prothrombin molecules (U-Protein Express BV Utrecht, the Netherlands). A hydroxyapatite column was used to obtain fully $\gamma$-carboxylated wild-type (WT) and mutant prothrombin. Prothrombin levels were measured by onestage clotting assay on an automated coagulation analyser (ACL 300 TOP) with reagents and protocols from the manufacturer (Instrumentation Laboratory, Breda, the Netherlands). At a final concentration of 90 $\mu \mathrm{g} / \mathrm{mL}$ in prothrombin deficient plasma, the recombinant WT and mutant prothrombins had prothrombin activity of $82 \%$ and $67 \%$, respectively. Furthermore, thrombin generation was performed using calibrated automated thrombography and Thrombinoscope software according to the manufacturer's instruction (Thrombinoscope BV, Maastricht, the Netherlands). Recombinant WT or p.Arg173Trp prothrombin was added to prothrombin deficient plasma (Siemens) at a final concentration of $90 \mu \mathrm{g} / \mathrm{mL}$ in the absence or presence of $0.1 \mathrm{U} / \mathrm{mL}$ unfractionated heparin (LEO Pharma BV) and in vitro thrombin generation was assessed. Figure 1E depicts representative thrombin generation curves. In the absence of unfractionated heparin, plasma containing p.Arg173Trp prothrombin had a substantially higher endogenous thrombin potential compared to plasma to which WT prothrombin was added $(2,400 \pm 113$ vs. $797 \pm 42 \mathrm{nM}$ IIa*min, $P<0.0001, \mathrm{n}=3$ ). Addition of unfractionated heparin reduced thrombin generation in plasma containing the p.Arg173Trp prothrombin by only $7.3 \pm 3.9 \%$ compared to a $31.4 \pm 9.3 \%$ reduction in thrombin generation in plas- ma containing WT prothrombin $(P=0.01, \mathrm{n}=3)$.

Finally, we performed molecular dynamics in order to simulate the effect of the p.Arg173Trp mutation (Online Supplementary Data). The prothrombin (F2) gene mutation is located in the 170s loop, near the non-primed side of the active site (Figure 2A). The results of molecular dynamics are consistent with a conformational alteration in the non-prime side of the active site due to the repositioning of the Trp173 side chain to minimize its solvent accessibility by packing up against Trp215 (Figure 2B).

Venous thrombosis is a disorder in which genetic predisposition plays an important role. However, in a large portion of patients with VTE no genetic defect can be detected. Several aspects contribute to the missing heritability for VTE, including rare variants. To identify such variants we combined linkage analysis with WES and found a novel prothrombin (F2) gene mutation p.Arg173Trp.

This mutation is assumed to be rare as it was absent in two control groups, e.g. in 24 probands of families with unexplained thrombosis and 100 healthy volunteers without VTE (selection from GoNL; http://www. nlgenome.nl/) (data not shown). Furthermore, this mutation was not present in double stranded single nucleotide polymorphism (https://www.ncbi.nlm. nih.gov/snp) or HGMD (http://www. hgmd.cf.ac.uk/ac/index.php).

Prothrombin deficiency has long been considered to only be associated with bleeding tendency until Miyawaki et al. reported in 2012 on a family with dysprothrombinemia and venous thrombosis. ${ }^{3}$ This dysprothrombinemia was caused by a substitution of arginine to leucine at position 221a in the F2 gene (prothrombin Yukuhashi). ${ }^{3}$ In the following years, two additional mutations at position 221a were reported, e.g. prothrombin Belgrade $^{4}$ and prothrombin Padua 2..$^{5}$ Apart from prothrombin Belgrade, these mutations were all associated with a strong risk of VTE at a young age. ${ }^{3,5}$ The association with VTE was explained by antithrombin resistance caused by mutations at position $221 \mathrm{a}^{3-5}$

All carriers of the prothrombin (F2) gene mutation p.Arg173Trp, with the exception of 216209 have had venous thrombosis. One family member who did not carry the mutation (216418) also had a history of DVT. We cannot exclude that additional unknown thrombophilic factors are present in the family. We have also observed in studies of protein C, S and antithrombin deficiency that non-affected family members have a higher
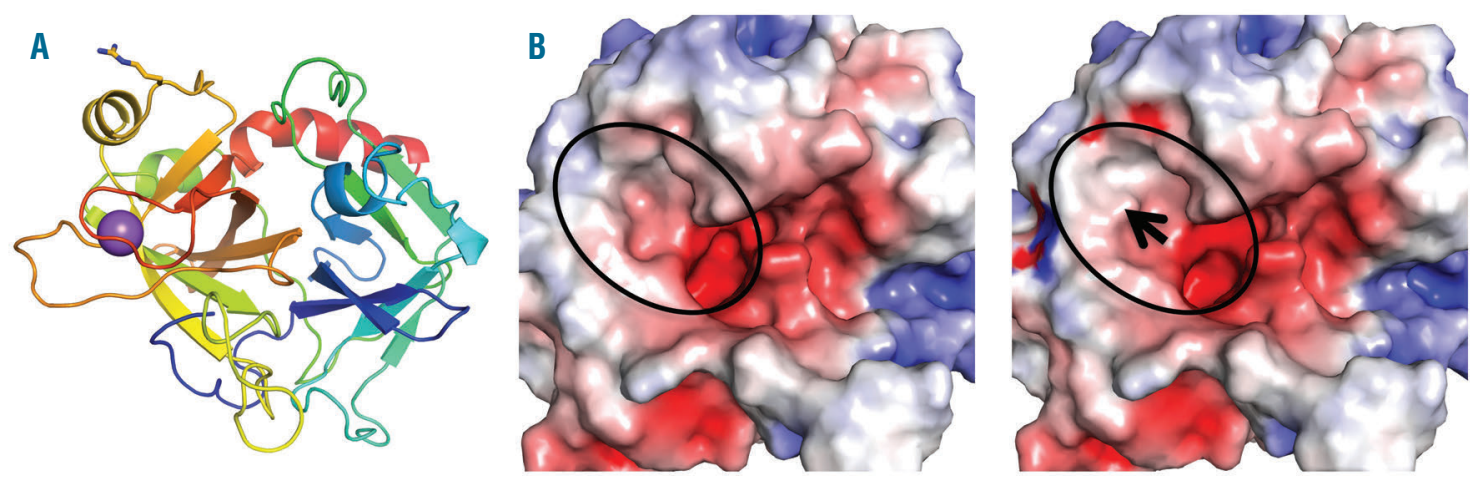

Figure 2. Structural characterization of the novel prothrombin (F2) gene mutation. (A) A ribbon diagram of the heavy chain of thrombin in the standard orientation showing the position of the mutation (side chain of Arg173 as sticks) and the $\mathrm{Na}^{+}$(purple ball) binding site (colored from $\mathrm{N}$-to-C terminus, blue to red). (B) Surface representation of the resulting structures after molecular dynamics simulation of wild-type (WT) (left) and the p.Arg173Trp mutant variant (right) thrombin coloured according to electrostatics. The oval indicates the non-primed side of the active site cleft, and the arrow indicates the position of the mutation. 
incidence of VTE than the normal population. ${ }^{6}$ However, venous thromboembolism is a common disorder and this one case in a family member without the mutation might also have been due to chance.

Our functional studies showed a clear heparin resistance that is suggestive for antithrombin resistance. In line with this, Tamura et al. performed an in vitro exploratory study using prothrombin mutants and showed that a recombinant p.Arg173Trp mutation exhibited a mild antithrombin resistance.

The side chain of p.Arg173 is not fully modeled in the structure of the antithrombin-thrombin Michaelis complex, suggesting that it is not making a single distinct contact with antithrombin. It is in a position where it could exert a favorable long-range electrostatic influence on docking, since it is in the vicinity of glutamates 232,377 and 378 on antithrombin. However, such an influence would be overwhelmed by the magnitude of the effect of heparin on the association rate. The heparin resistance of the p.Arg173Trp thrombin variant therefore suggests something more profound than the loss of a favorable interaction or electrostatic influence. A structural rearrangement of the active site caused by the introduction of a large hydrophobic residue to a solvent-exposed loop is more likely (Figure 2A). The 170s loop has been implicated in the zymogen to protease conformational change and allosteric activation. The molecular dynamics results suggest a distinct conformational change in the non-prime side of the active site due to the Trp173 burying itself as much as possible thereby interfering with antithrombin inhibition (Figure 2B). However, more evidence is still needed to conclude that the prothrombin mutation p.Arg173Trp leads to antithrombin resistance.

Finally, at a final concentration of $90 \mu \mathrm{g} / \mathrm{mL}$ in deficient plasma, the recombinant WT and mutant showed borderline low prothrombin activity levels, which is in contrast with the increased thrombin potential observed with the thrombin generation assay. Such a discrepancy has also been observed for four antithrombin-resistant mutations in position 221a. This discrepancy could be explained by the fact that clotting assays measure only the clotting time corresponding to the initiation phase of the coagulation process, whereas thrombin generation assays also provide information about the amplification and propagation phases of the hemostatic system.

In conclusion, in this study we identified a rare novel prothrombin (F2) gene mutation p.Arg173Trp that causes heparin resistance which is suggestive for antithrombin resistance. Evidently further investigations are needed to fully disentangle the mechanism responsible for the potential association between the prothrombin (F2) gene mutation p.Arg173Trp and venous thrombosis.

René Mulder, ${ }^{1}$ Ton Lisman, ${ }^{2}$ Joost C.M. Meijers, ${ }^{3}$

James A. Huntington, ${ }^{4}$ André B. Mulder' and Karina Meijer ${ }^{5}$

${ }^{1}$ Department of Laboratory Medicine, University of Groningen, University Medical Center Groningen, Groningen, the Netherlands; ${ }^{2}$ Surgical Research Laboratory and Section of Hepatobiliary Surgery and Liver Transplantation, Department of Surgery, University of Groningen, University Medical Center Groningen, Groningen, the Netherlands; ${ }^{3}$ Department of Molecular and Cellular Hemostasis, Sanquin Research, Amsterdam and Amsterdam UMC, University of Amsterdam, Department of Experimental Vascular Medicine, Amsterdam Cardiovascular Sciences, Amsterdam, the Netherlands; ${ }^{4}$ Department of Haematology, Cambridge Institute for Medical Research, University of Cambridge, Cambridge, UK and 'Department of Hematology, University of Groningen, University Medical Center Groningen, Groningen, the Netherlands

Correspondence: RENÉ MULDER - r.mulder01@umcg.nl.

doi:10.3324/haematol.2019.232504

Information on authorship, contributions, and financial 2 other disclosures was provided by the authors and is available with the online version of this article at wWw. haematologica.org.

\section{References}

1. Morange PE, Tregouet DA. Deciphering the molecular basis of venous thromboembolism: where are we and where should we go? Br J Haematol. 2010;148(4):495-506.

2. McCarthy MI, Abecasis GR, Cardon LR, et al. Genome-wide association studies for complex traits: consensus, uncertainty and challenges. Nat Rev Genet. 2008;9(5):356-369.

3. Miyawaki Y, Suzuki A, Fujita J, et al. Thrombosis from a prothrombin mutation conveying antithrombin resistance. $N$ Engl J Med. 2012;366(25):2390-2396.

4. Djordjevic V, Kovac M, Miljic P, et al. A novel prothrombin mutation in two families with prominent thrombophilia--the first cases of antithrombin resistance in a Caucasian population. J Thromb Haemost. 2013;11(10):1936-1939.

5. Bulato C, Radu CM, Campello E, et al. New Prothrombin mutation (Arg596Trp, prothrombin Padua 2) associated with venous thromboembolism. Arterioscler Thromb Vasc Biol. 2016;36(5):1022-1029.

6. Mahmoodi BK, Brouwer JL, Ten Kate MK, et al. A prospective cohort study on the absolute risks of venous thromboembolism and predictive value of screening asymptomatic relatives of patients with hereditary deficiencies of protein $\mathrm{S}$, protein $\mathrm{C}$ or antithrombin. J Thromb Haemost. 2010;8(6):1193-1200.

7. Tamura S, Murata-Kawakami M, Takagi Y, et al. In vitro exploration of latent prothrombin mutants conveying antithrombin resistance. Thromb Res. 2017;159:33-38. 